\title{
Model of Plant Productivity and a Computer System for Optimization of Agro-Technology Using the Method of Exergic Analysis
}

\author{
V. A. Mudrik \\ Institute of Basic Biological Problems, Russian Academy of Sciences, Pushchino, Moscow Region, Russia \\ Email: vilenmudrik@gmail.com
}

How to cite this paper: Mudrik, V.A. (2017) Model of Plant Productivity and a Computer System for Optimization of Agro-Technology Using the Method of Exergic Analysis. World Journal of Engineering and Technology, 5, 12-17. https://doi.org/10.4236/wjet.2017.55B002

Received: July 21, 2017

Accepted: November 14, 2017

Published: November 17, 2017

\begin{abstract}
A model of a potentially effective type energy-resource-saving of optimization of agro-technologies, based on the principle subordination of synergetics, was established. There was developed computer system energy-resource-saving optimization of agricultural technologies. The main feature of crop production is provided by the plants which themselves are self-organizing organisms. This allows us to adopt the principle of subordination of synergetics as the basis of the model. The value of free energy at the input into plants, estimated by the process of photosynthesis, is equal to the value of "radiation exergy for plant growth". Assessment of the use of radiation exergy is carried out based on the energy-converting characteristics of plants, which were obtained in climate chambers under controlled conditions. We used the model based on the principle of subordination of synergetics to develop common quantitative mutually agreed definitions of the main agroecological variables: Agroclimatic and Meliorative potentials of lands, their fertility, and potential (maximum) productivity of plants under different environment conditions.
\end{abstract}

\section{Keywords}

Solar Radiation, Exergy of Solar Radiation, A Model Plant Productivity, A Computer System of Agrotechnology Optimization

\section{The Principle of Subordination of Synergetics}

We have developed a potentially effective type of dynamic model of plant productivity [1] [2] [3] [4]. The most progressive direction of modern fundamental science is self-organization [5], which is sometimes called synergetics. Synergetics is a special case of self-organization. An important principle of synergetics is 
the principle of subordination of synergetics. The essence of this principle is as follows. When analyzing complex systems with many variables, the one that most strongly affects the operation of the system is selected. This variable is called an order variable. In the further analysis of a complex system, it is considered only as a variable of order. All other variables can be taken into account in the analysis as control parameters. The transformation of energy by living organisms is carried out based of biochemical or photophysical processes. The main process of biological transformations of energy in plants is the photosynthesis of plants. In connection with this, it is advisable to begin the assessment of the bioconversion of energy in plants by determining the potential transformability of the energy of solar radiation in the process of photosynthesis-the exergy of the energy of solar radiation with respect to photosynthesis. As an initial value for the quantitative determination of agroecological variables, the exergy (working capacity in relation to the process of photosynthesis) of solar energy is accepted. The value of exergy limits the maximum value of both the fertility of the land area and the potential productivity (species, variety) of plants under given ecological conditions.

In crop production more than $96 \%$ of the energy involved in the process is solar radiation, and only $2 \%-3 \%$-technogenic energy [1] [2]. The primary transformation of the energy of solar radiation is possible only by autotrophic photosynthesizing plants. In the crop model, the radiation exergy is taken as the reference point (the beginning of the calculation) - the portion of energy of solar radiation potentially suitable for photosynthesis and the formation of productivity $\left(e_{S R}\right)$.

\section{The Spectral Efficiency of Photosynthesis-K( $-K) p h$}

The process of photosynthesis is selective to radiation of different wavelengths. In accordance with the law of quantum equivalence, the effect of converting the radiation energy is proportional to the number of effectively absorbed photons. The absorption and conversion of radiation energy by plants in the process of photosynthesis is taken into account on the basis of the experimentally established spectral efficiency of photosynthesis, $K(\lambda) p h$. The values of $K(\lambda) p h$ are determined at the irradiance values of the unsaturated photoelectric effect and are expressed in relative units. The relative spectral efficiency of photosynthesis $K(\lambda) p h$ is the same for all chlorophyll-containing plants. To obtain absolute values of $K(\lambda) p h$, the spectral efficiency of photosynthesis is determined for a wavelength $\lambda=680 \mathrm{~nm}$ corresponding to the maximum spectral efficiency of photosynthesis. We have jointly carried out a statistical analysis of 66 experimental spectra of photosynthesis by six authors (Figure 1, curve 2) [1] [2] [3] [4] [7]. Values of the exergy of solar radiation with respect to photosynthesis can be determined by the integration of the spectrum of solar radiation $\left(\varphi(\lambda)_{s}\right)$, as well as radiation of any other spectral composition, multiplied by the spectral efficiency (action spectrum) of photosynthesis $\left(K(\lambda)_{p h}\right)$ [1] [2] [3] [4]. 


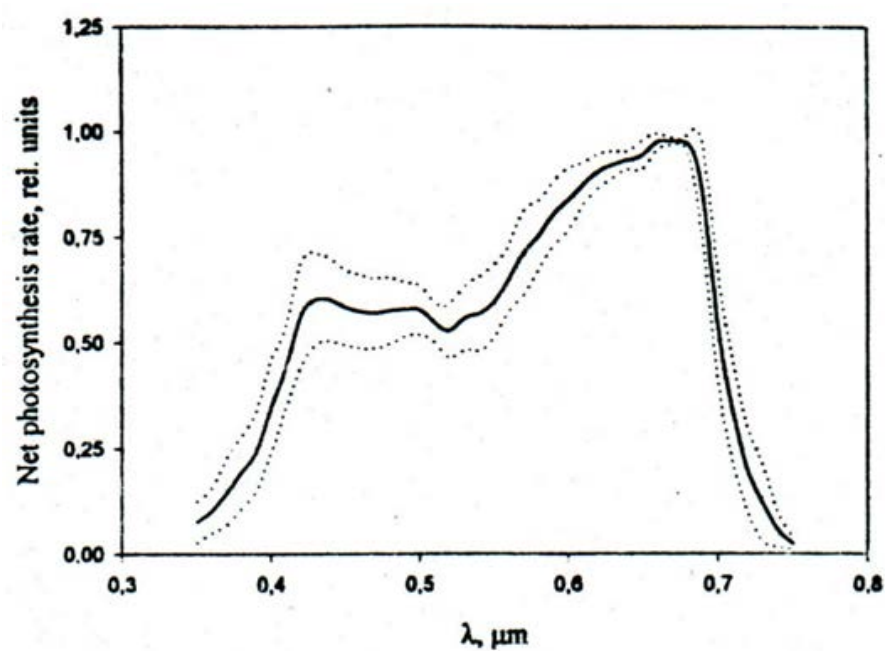

Figure 1. Average normalized action spectrum of photosynthesis (-) according to 66 experimental data by 6 authors [1] [2] [3] [4] for different types of plants, and the respective 95\% confidence intervals ( $\cdots)$.

$$
e_{S R}=g \times \int_{\lambda 1}^{\lambda 2} \varphi(\lambda)_{s} \times K(\lambda)_{p h} \mathrm{~d} \lambda
$$

where $g$ is the maximum quantum efficiency of photosynthesis at a peak of action spectrum at $680 \mathrm{~nm} . \lambda_{1}=300 \mathrm{~nm}, \lambda_{2}=750 \mathrm{~nm}$, the spectrum of solar radiation $\left(\varphi(\lambda)_{s}\right)$.

The value of the exergy of solar radiation for a given period of time $\left(\Delta e_{S R}\right)$ is determined from the data from the meteorological stations or by direct measurements of this value by the exergy measuring device with a spectral sensitivity, similar to the spectrum of photosynthesis $K(\lambda)_{p h}$.

The spectral efficiency of photosynthesis $\left(K(\lambda)_{p h}\right)$, averaged from the data of 66 action spectra. We have jointly carried out a statistical analysis of 66 experimental spectra of photosynthesis by six authors (Figure 1, curve 2) for different plants, is shown in Figure 1 [1] [2] [3] [4].

The solar exergy $\left(\mathrm{e}_{\mathrm{SR}}\right)$ is the theoretical limit (point of reference) of both the productivity of the crops, and the fertility of the land. All other factors $(f)$ in the model are taken into account as controlled parameters, each with its own optimality coefficient $\left(K_{f_{-} O P T}\right)$. The efficiency of using the solar energy in real conditions by plants of different species (varieties, hybrids) depends both on their intrinsic properties (characteristics) and on the changing environmental factors and soil properties. The values of the coefficients of optimality of ecological and other factors $K_{f}$ taken into account are determined as follows:

$$
K_{f}=I_{f_{-} R E A L} / I_{f_{-} O P T}
$$

where $I_{f_{-} R E A L}$ is the rate of photosynthesis of plant of this species (variety, hybrid) under current value of the factor f; $I_{f_{-} O P T}$ is the same rate under optimal (for photosynthesis) value of this factor. Measurement of the exergy of optical radiation was carried out with the help of exergy sensors of solar radiation, whose 
spectral sensitivity is similar to the spectrum action of photosynthesis. The application of the thermodynamic index (the water potential of soils) makes it possible to choose for different soils a property that determines the availability of water for plants. Therefore, the moisture content of plants can be determined reliably only by the water potential of the soil.

Knowing energy-transforming characteristics of plants makes it easier to zone and introduce plants, taking into account the effects of climatic factors and soil properties on the effectiveness of use of solar energy by plants, and to adjust the technological process for improving its efficiency. These studies were carried out in climatic chambers with controlled environmental parameters (Figure 2) [6] [7] [8] [9].

For the greatest majority of agricultural plants air temperature and soil humidity were the main climatic factors. For some plants (wheat, cucumbers, tomatoes, corn) we obtained of the energy-transforming characteristics for air temperature and soil humidity [6] [7] [8] [9]. It is advisable to put these energy-transforming characteristics of plants in the passports of corresponding varieties and hybrids. This information will make it easier to identify the ability to grow new crops not only in the zones for which they are zoned, but also in other regions. Knowing energy-transforming characteristics of plants makes it easier for zonning and introducing plants, taking into account the effects of climatic factors and soil properties on the effectiveness of use of solar energy by plants. Knowledge of energy-transforming characteristics is necessary for correcting the technological process to improve its efficiency. To find the exergy of agroclimatic potential of photosynthesis under current conditions, we compare the values of the coefficients of optimality of the factors, and select the limiting factor, which at a given time is at a relative minimum $\left(K_{f_{-} \min }\right)$. The solar radiation for a period of time $\Delta t\left(\Delta e_{S R}\right)$ multiplied by the coefficient of optimality of the factor that has the lowest value in this period $\left(K_{f_{-} \min }\right)$ is equal to the exergy of the agroclimatic potential $\left(\mathrm{e}_{A P}\right)$ for this time period

$$
e_{A P}=\Delta e_{S R} \times K_{f_{-} \min }
$$

It is one of the main characteristics of agricultural lands and can be correctly

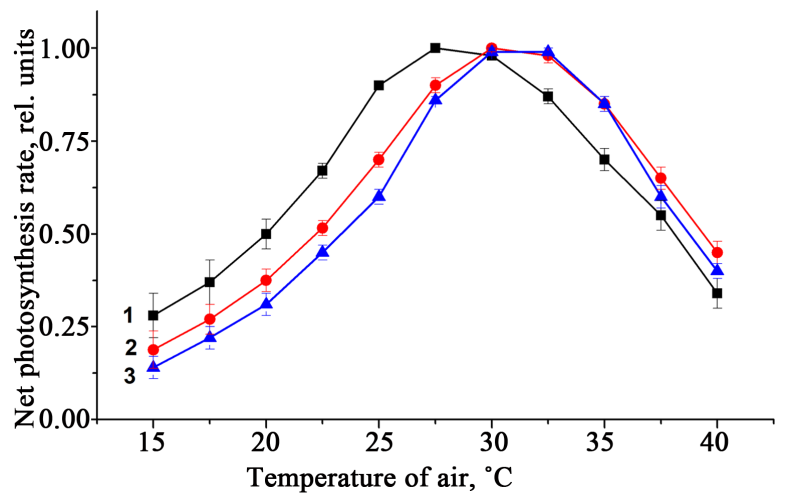

Figure 2. Dependence of the relative rate of photosynthesis of corn on air temperature at 
a total irradiance of $200(1), 300$ (2) and 500 (3) W/m² (corresponding to 40, 60, and 100 $\mathrm{W} / \mathrm{m}^{2}$ exergy of solar radiation).

quantitatively determined, taking into account changing climatic factors. Using the above formula one can also determine the fertility of the land by adding the coefficients of optimality of various soil properties. We used the model based on the principle of subordination of synergetics to develop common quantitative mutually agreed definitions of the main agroecological variables: Agroclimatic and Meliorative potentials of lands, their fertility, and potential (maximum) productivity of plants under different environment conditions.

Until now, in the management of the production process of organisms, the genetic information of organisms are not directly taken into account, obviously because of the lack of data on the identification of genetic structures with the ecological and physiological properties of organisms. The possibility of such identification has already appeared and it becomes necessary to directly take into account in the management of the production process genetic information of producers. Obviously, the structures of genetic information contain elements that reflect the properties of the ideal orientation of progressive evolution [9]. In selection, seed and especially in biotechnologies, it is clearly necessary to control not only the availability, but also the quantitative evaluation of these elements of the genetic information structures of agricultural organisms.

\section{References}

[1] Sventitskiy, I.I., Kasumov, N.E., Mudrik, V.A., Alkhazova, E.O. and Palagin, A.V. (2013) Method and Computer System of Energy-Resource-Saving Optimization of Agricultural Technologies and Assessment of Their Effectiveness Level. VIESH, Москва, 64 p. (In Russian)

[2] Sventitsky, I., Kasumov, N. and Mudrik, V. (2013) Exergetic, Energy-Informational Theorization of Agrarian-Ecological Knowledge. Vestnik VIESH, 3, 10-19. (In Russian)

[3] Sventitsky, I.I., Korolev, V.A. and Mudrik, V.A. (2010) Optimum Management in Agricultural Production and Nature Management on a Self-Organizing Basis. In: Mathematical Models and Information Technologies in Agricultural Biology: Results and Perspectives. AFI, St. Peterburg, 40-43. (In Russian)

[4] Mudrik, V.A., Sventitsky, I.I. and Grishin, A.P. (2010) Synergetic Model and a System of Multiply Connected Microclimate Management for Greenhouses. In: Mathematical Models and Information Technologies in Agricultural Biology. Results and Perspectives, AFI, St. Peterburg, 40-43. (In Russian)

[5] Strebkov, D.S., Sventitskiy, I., Zhmakin, I., Korolev, V. and Mudrik, V.A. (2010) The Optimum Management in the Agrarian Production and Nature-Use on the Self-Organization Base. Collection of Abstracts from 4 rd International Conference TAE 2010, Czech University of Life Sciences Prague, Prague, 598-602.

[6] Mudrik, V., Knyazeva, I., Kosobrukhov, A. and Pigulevskaya, T. (2003) Changes in the Photosynthetic Characteristics of Plantago major Plants Caused by Soil Drought Stress. Plant Growth Regulation, 40, 1.

[7] Mudrik, V., Stoyanov, P. and Ivanov, B.N. (2000) Evaluation of Maize Productivity Considering Solar Energy Use Limitation by Environmental Factor. Photosynthesis 
Research, 66, 177-187.

[8] Mudrik, V.A., Romanova, A.K., Ivanov, B.N., Novichkova, N.S. and Polyakova, V.A. (1997) Effect of Increased $\mathrm{CO}_{2}$, Concentration on Growth, Photosynthesis, and Composition of Pisum sativum L. Plants. Russian Journal of Plant Physiology, 44, 141-146.

[9] Mudrik, V.A. and Vil'chek, G.E. (2001) Ecophisiological Responses of Larix sibirica Ledeb, and Pinus sibirica Du Tour Undegrowth to Climate Change. Russian Journal of Ecology, 32, 243-248.

[10] Kasumov, N., Sventitsky, I. and Mudrik, V. (2013) The Anthropic Principle as Consequence of Progressive Evolution and Level of Its Precision. Research in Agricultural Electric Engineering, 3, 108. 